\title{
EL PANTOCRÁTOR DEL LIBER SANCTI ANDREAE DE CASTELLO: UN ESTUDIO DEL FOLIO 2R DEL MANUSCRITO MS528 DE CAMBRAI
}
O PANTOCRATOR DO LIBER SANCTI ANDREAE DE CASTELLO: UM ESTUDO DO FÓLIO 2R DO MANUSCRITO MS528 DE CAMBRAI

\section{THE CHRIST PANTOCRATOR OF LIBER SANCTI ANDREAE DE CASTELLO: A STUDY OF FOLIO 2R OF MANUSCRIPT MS528 OF CAMBRAI}

Fidel Pascua Vílchez ${ }^{1}$

RESUMEN: descripción y análisis del folio 2-recto del manuscrito inédito del siglo XII Ms528 de Cambrai (Francia). Con base en el propio manuscrito, en Sánchez Prieto (2015), Godoi (2014) y Gibbs (2012), entre otros, se describe y analiza: la iluminación del pantocrátor, la anotación de la biblioteca, la numeración del folio, una poesía en latín escrita al margen derecho, una anotación escrita por un bibliotecario a finales del siglo XVIII y una operación matemática referente a las fechas. El análisis permitió determinar los lugares en los que estuvo depositado el manuscrito, las diferentes organizaciones del códice, el nombre de un copista y las fechas clave en su elaboración. Concluimos que: el manuscrito tuvo tres sedes distintas; fue elaborado entre 1115 y 1170; es necesario establecer una nueva numeración del códice.

PALABRAS CLAVE: Liber sancti Andreae de castello; manuscrito iluminado; santa Maxelendis; Abbaye Saint-Sépulcre de Cambrai; Abbaye Saint-Andre du Cateau.

RESUMO: descrição e análise do fólio 2-recto do manuscrito inédito do século XII Ms528 de Cambrai (França). Com base no próprio manuscrito, em Sánchez Prieto (2015), Godoi (2014) e Gibbs (2012), entre outros, é descrito e analisado: a iluminação do pantocrator, a anotação da biblioteca, a numeração do fólio, uma poesia em latim escrita na margem direita, uma anotação escrita por un

\footnotetext{
*Este artigo é proveniente de um projeto de pesquisa cadastrado na PRPPG da UNILA intitulado "O diálogo entre texto e imagem na história do homiliário Saint-Andre du Cateau Ms528 de Cambrai", sem financiamento.

${ }^{1}$ Doutor em Estudos da Linguagem pela Universidade Estadual de Londrina (UEL). Professor de Latim e de Língua Espanhola na Universidade Federal da Integração Latino-Americana (UNILA). Telefone de contato: (45) 99137-1554. E-mail: fidel.vilchez@ unila.edu.br
} 
bibliotecario a finales del siglo XVIII e uma operação matemática em relação às datas. A análise permitiu determinar os lugares nos quais o manuscrito ficou depositado, as diferentes organizações do códice, o nome de um copista ou iluminador e as datas-chave na sua elaboração. Conclui-se que o manuscrito teve três sedes diferentes ao longo dos séculos; foi elaborado entre 1115 e 1170; é preciso estabelecer uma nova numeração do códice.

PALAVRAS-CHAVE: Liber sancti Andreae de castello; manuscrito iluminado; santa Maxelendis; Abbaye Saint-Sépulcre de Cambrai; Abbaye Saint-Andre du Cateau.

ABSTRACT: description and analysis of folio 2-recto of unpublished $12^{\text {th }}$ century manuscript of Cambrai (France). Based on the manuscript itself, on Sánchez Prieto (2015), Godoi (2014) and Gibbs (2012), among others, it is described and analysed: the Christ Pantocrator illumination, the library's notation, the folio number, a poetry in Latin written in the right margin, a notation written by a librarian at the end of $18^{\text {th }}$ century, a mathematical operation related to dates. The analysis allowed to establish the diferent places the manuscript was set, the diferent organisation of codex, the copyist or illuminator one's name and key dates for its creation. It is concluded that: the manuscript had three diferent locations over the centuries; it was drawn up between 1115 and 1170; its necessary to establish a new codex numbering.

KEYWORDS: Liber sancti Andreae de castello; Illuminated manuscript; saint Maxelendis; Abbaye Saint-Sépulcre de Cambrai; Abbaye Saint-Andre du Cateau.

\section{Introducción}

El Liber sancti Andreae de castello es un códice iluminado inédito del siglo XII, depositado en la Bibliothèque Municipale de Cambrai (Francia) y catalogado como Manuscrit Ms528, con el título de Homiliarium. Según la ficha de la biblioteca, está constituido por 274 folios de pergamino encuadernados en cuero, sus dimensiones son $445 \times 338 \mathrm{~mm}$ y está escrito íntegramente en latín.

Este manuscrito está digitalizado en su totalidad mediante escáner, en escala de grises y disponible en Internet, a través de la página web de libre acceso BVMM Bibliotèque Virtuelle des Manuscrits Médiévaux $<\underline{\text { http://bvmm.irht.cnrs.fr/> }}$. Por su parte, todas sus iluminaciones están digitalizadas y disponibles en color en el mismo site y también en la página web 
Enluminures, dependiente del Ministère de la Culture de Francia: $<$ http://www.enluminures.culture.fr/>, también de libre acceso, aunque su uso está sujeto a derechos de autor.

A pesar de que el Liber sancti Andreae de castello está catalogado como Homiliarium, menos de la mitad de su contenido se corresponde realmente con homilías, pues reúne, además de este tipo de texto, sermones, comentarios a libros del Antiguo Testamento, epístolas, comentarios a los Evangelios, tratados filosófico-religiosos diversos, un capítulo del Libro de los Salmos y una hagiografía.

En total, el códice presenta 196 textos diferentes, además de dos grandes iluminaciones al comienzo (la imagen del copista y el Pantocrátor). Tras analizar su contenido, hemos podido comprobar que nueve de ellos están formados por más de un texto; inclusive, por textos pertenecientes a distintos autores, lo que, en realidad, significa que el número total de textos diferentes en el Ms528 se eleva hasta los 223.

El contenido íntegro del Homiliarium se ofrece como Anexo.

El códice presenta una gran iluminación en su folio 2-recto, la cual sirve de decoración inicial del mismo: la imagen de un pantocrátor que ocupa toda la cara del folio, escoltado a la izquierda por el protoapóstol san Andrés y a la derecha por santa Maxelendis, una mártir de Cambrai del siglo VII. Completa la escena la figura de un monje llamado Rainerus, que, postrado en actitud suplicante, le agarra los pies a Cristo, con el rostro vuelto hacia san Andrés, pidiendo su intercesión (Figura 1): 
Figura 1: Folio 2-recto del Liber sancti Andreae de castello (f002r).

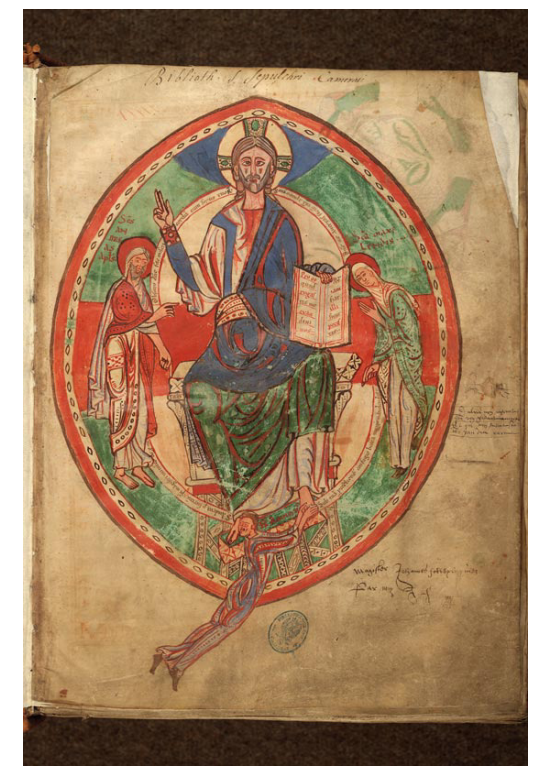

Fuente: Ministère de la Culture - Enluminures.

La imagen del pantocrátor, junto a la de los otros tres personajes que lo acompañan dentro de la mandorla, destaca por su hermosura, su magnificencia y es el elemento principal que sobresale en el recto del folio, pero no es el único presente. Hay otros elementos añadidos con posterioridad a la elaboración de la imagen que aportan datos sobre los lugares en los que estuvo depositado el manuscrito, la organización de las páginas, la época en que fue escrito, la época en que fue revisado o catalogado y la identidad del revisor/catalogador. Además de eso, el folio incluye en su parte central derecha una poesía escrita en latín.

Por todo lo dicho, los elementos incluidos en el recto del folio 2 del manuscrito Ms528 de Cambrai tienen singular importancia para entender el conjunto del códice. Tratándose, además, de un manuscrito inédito, resulta pertinente hacer su análisis y divulgación de resultados, para que sirva también de base a futuras investigaciones sobre el mismo.

La anotación en la parte superior y las distintas sedes del manuscrito 
El primer elemento que aparece en el folio, según se observa de arriba abajo, es una anotación en latín en la parte central superior, en letra cursiva y de cuidada caligrafía, que apunta a uno de los lugares en los que estuvo depositado el manuscrito: "Biblioth. S. Sepulchri Camerai" (Biblioteca del Santo Sepulcro de Cambrai), lo que da pie a hacer memoria de la historia del códice:

El manuscrito debe su nombre al título que el último de los copistas le asignó a su conclusión en el verso del último folio, liber sancti Andree de castello, en una mezcla de latín y francés medieval.

Ese colofón anotado al final del códice nos informa de que fue elaborado/copiado en la Abbaye de Saint-André du Cateau (Abbatia Sancti Andreae de Castello en latín), una abadía benedictina fundada en el siglo XI, situada en la actual comuna francesa de Le Cateau-Cambrésis, a veinticinco quilómetros de la ciudad de Cambrai, en el nordeste de Francia. Según Godoi (2014, p430), esta abadía fue consagrada por Gerard I, Obispo de Cambrai, alrededor del año 1020, y en el año 1048 la confirmó Henrique III, emperador del Sacro Imperio Romano Germánico.

Por su parte, Bruzen de la Martinière (1768: 218) dedica en su Le grand dictionnaire géographique, historique e critique una entrada a la Abbaye SaintAndre:

La Abadía de san Andrés, abadía de la Orden de san Benito, situada en Le Cateau-Cambrésis: la opinión común es que Gerardo I, obispo de Cambrai da comienzo a este monasterio en el año 1020 y que la iglesia fue consagrada al año siguiente en presencia de muchos obispos, condes y señores vecinos. El mismo obispo confirma todas las limosnas que le habían sido hechas por una carta del año 1026. El emperador Conrado se las confirma también en 1033. Nicolás, obispo de Cambrai, confirma de nuevo en el año 1156 todas las limosnas y donaciones hechas a la mencionada abadía por diversos obispos $y$ señores en varias ocasiones (MARTINIÈRE, 1768: 218, traducción propia).

Con todo, Martinière se apoya en la obra de un autor anterior, Histoire de Cambray e du Cambrésis, de Jean-Baptiste Carpentier Carpentier (1664), que no deja de ser otra fuente secundaria que bebe de fuentes anteriores. Concretamente, los dos textos de referencia sobre la época de fundación de la 
abadía son el Andreas castri Cameracesii chronicon y Gesta Pontificorum Cambriacensium.

El Andreas castri Cameracesii chronicon es una crónica de la abadía escrita por cierto monje anónimo de la misma que acompañó y aconsejó al obispo Walcher en el Concilio de Clermont-Ferrand de 1095. Dicha crónica, escrita en 1133, describe en tres libros la historia del monasterio y de la región de Cambrai. Fue recopilada en 1841 por Ludwig Bethmann y publicada posteriormente en 1853 :

Entonces, el señor obispo, dispuesto al cumplimento de la promesa que antes había concebido en su ánimo, construyó con eficaz trabajo un oratorio en honor de san Andrés, el apóstol y amigo de Dios, al cual este se encomendaba atentamente antes que a los demás santos, y transfirió allí a veinticuatro monjes que habían de servir bajo la sagrada religión y la norma regular, y les puso como abad a su hermano Elberto, un hombre de vida venerable (BETHMANN, 1853: 246, traducción propia).

Por su parte, el Gesta Pontificorum Cameracensium es un texto de autor desconocido que narra los hechos relacionados con los obispos de Cambrai entre los años 1041 y 1179. Al comienzo de la obra, hace un repaso de la historia y personalidades ilustres del lugar y se menciona la construcción de un oratorio a san Andrés dentro del castillo de santa María, entre los años 1023 y 1024:

También la cuarta parte del tesoro público de Péronne, que había adquirido de cuatro hombres ingenuos (de Roberto, naturalmente, y de Hubaldo, Atelino y Suevo) la destinó de la mesa del obispo para el castillo de santa María, que está al lado. Allí mismo construyó el oratorio de san Andrés desde sus cimientos, y la tercera parte de la cuarta parte del tesoro público citada anteriormente la destinó a la congregación de monjes, que con pía disposición fomentó mientras vivió (ANÓNIMO, 1853: 168, traducción propia).

Sin embargo, la anotación de la parte superior del folio demuestra que el manuscrito fue a parar a la biblioteca de la Abbaye du Saint-Sépulcre (Abadía del Santo Sepulcro) de Cambrai, un monasterio benedictino creado por el obispo Lietberto en 1064, después de un fallido intento de peregrinación a Tierra Santa: 
El obispo don Lietberto regresó de un viaje que no llegó a completar, en el que ansiaba llegar a Jerusalem, como se dijo antes, pero habiendo surgido diversos peligros que se lo impidieron, fue zarandeado por tierra y mar y se vio obligado a dar marcha atrás, pero se propuso lograrlo de otra manera: edificar fuera de la muralla de Cambrai una iglesia, naturalmente en honor del Santo Sepulcro, como se la conoce hasta hoy. Y lo que concibió con su egregia mente lo llevó a cabo hasta el final devotísimamente, pues construida apropiadamente, siendo traídos de fuera los cuerpos de muchos santos, la consagró solemnemente el año de Dios de 1064, poniendo a su servicio al abad don Waldrico y estableció allí a monjes al servicio de Dios, así como a mujeres que velasen fuera el sepulcro de Cristo. Y, ciertamente, hizo que fuese preparado también para sí en la misma iglesia un sepulcro en el que también fue enterrado (ANÓNIMO, 1853: 264, traducción propia).

La abadía sufrió diversas vicisitudes a lo largo de los siglos. Se mantuvo como monasterio hasta el 15 de junio de 1791, año en el que fue clausurado por motivos relacionados con la Revolución Francesa. La iglesia de la abadía es la actual Cathédral Notre-Dame de Grâce de Cambrai (Catedral Nuestra Señora de Gracia de Cambrai) desde 1804. El acervo de su biblioteca pasó a la Bibliothèque Municipale de Cambrai, incluido el Ms528.

\section{La numeración del folio y la organización del códice}

El segundo elemento que encontramos en el recto del folio es su numeración en la parte superior derecha; concretamente, un número 2, en símbolo arábigo, tal y como lo escribimos hoy en día. Es decir, para el bibliotecario o catalogador que revisó el códice y lo numeró, este folio era el segundo.

Esta numeración permite hacer una reflexión sobre las dos numeraciones distintas que contiene el manuscrito y, como consecuencia de ello, sobre la diferente organización del códice a lo largo del tiempo:

Efectivamente, todos los folios del manuscrito contienen, en la parte superior derecha del recto, un número en caracteres arábigos ordenados desde el 1 hasta el 275. Además, la Bibliothèque Municipale de Cambrai informa que 
faltan dos folios: el 176 y el 177: "Folio manquant 176, 177', hecho que se puede comprobar siguiendo el orden de la numeración. Esto permite deducir que existe un error en la catalogación actual de la Bibliothèque Municipale de Cambrai, porque si el total de folios era 275 y faltan 2, entonces el manuscrito contiene 273 folios y no 274.

Ahora bien, al revisar el final del folio 175 y el comienzo del 178, para conocer cuál era el tamaño de la laguna del texto que faltaba por la ausencia de los dos folios, comprobamos que no existía tal laguna, pues el folio 175 termina con la oración: Illic iter est laudis ad aeternam laudem sine fine mansuram, sed nemo apraehendet illud, nisi ego praemonstravero; y, por su parte, el folio 178, el siguiente en el códice, comienza con: Propterea carissimae quia iter salutis nostrae in laudibus est Salvatoris, hortor vos et commoneo in hac sacra sollempnitate Genitricis Dei Mariae nolite cessare.

Al unir los dos fragmentos se puede comprobar, efectivamente, que no existe salto o laguna en el texto; este se corresponde con el Capítulo VI de Epistula IX: ad Paulam et Eustochium. De assumptione beatae Mariae Virginis (Epístola 9: a Paula y Eustoquio, sobre la Asunción de santa María Virgen) de Jerónimo de Estridón (san Jerónimo):

Allí está el camino de alabanza que conduce a la eterna alabanza que ha de permanecer sin fin, pero nadie lo tomará a no ser que yo lo indique antes. Por este motivo, queridísimas, porque el camino de nuestra salvación está en las alabanzas al Salvador, os exhorto y os advierto para que no dejéis de hacerlas en esta sagrada celebración de María, la Madre de Dios (JERÓNIMO DE ESTRIDÓN, 1846: 132, traducción propia).

Sucede que, además de esta numeración moderna, existe otra numeración anterior en caracteres romanos, situada en la parte superior central del verso de los folios, que no coincide con la otra:

De los 273 folios de pergamino que conforman el códice (y no 274, como indica la ficha de la biblioteca), 271 están ordenados en números romanos, desde el $i$ hasta el cclxxiii en la parte superior central del verso de cada folio, con la particularidad de que hay dos saltos en la numeración: no existe el número de folio $c i$ (pues se pasa directamente del $c$ al $c i i$ ), ni tampoco 
el $c x l$, porque, del mismo modo, hay un salto del cxxxix al $c x l i$, aunque no existe laguna en el texto en ninguno de los dos casos, como pudimos comprobar:

En efecto, las tres últimas líneas del folio numerado en el verso como $c$, que dan inicio a la oración: "Qui fabulis otiosis obscenisve carminibus vel detractionibus aurem libenter aperit, hanc animae suae" tienen continuidad en el recto del folio siguiente: "portam mortis efficit", numerado, a su vez, como cii en su verso. El texto en cuestión es Homilia XV. In dominica decima sexta post Trinitatem, del tercer libro de homilías de Beda el Venerable (homilías atribuidas a Beda, no genuinas), según la catalogación y edición de las homilías de Beda que J. P. Budé hizo en 1950, aunque en otros repertorios figura como la Homilia XIV:

El que con agrado presta oídos a relatos ociosos, a poemas obscenos o a comentarios despreciativos obtiene como resultado esta puerta de muerte para su alma" (BEDA, 1850: 299, traducción propia).

Esto indica que el salto en la numeración tampoco se debe a la ausencia del folio.

Del mismo modo, las dos últimas líneas del folio numerado originalmente en el verso como cxxxix se corresponden con el comienzo de la siguiente oración: "Genas quippe ut turturis habet anima quae ab omni impudicitiae peste", la cual tiene continuidad en el recto del folio siguiente: "castam se custodit et immunem", numerado en su verso como cxli. En este caso, el texto del que forma parte la oración completa es Homilia XV. In purificatione beatae Mariae, del primer libro de homilías de Beda el Venerable (homilías genuinas de Beda): "Puesto que tiene ojos como de paloma el alma que se mantiene casta e inmune de todo mal de impudicia” (BEDA, 1850: 80).

Tampoco se puede decir, en este caso, que el salto en la numeración se deba a la ausencia del folio.

En relación a la numeración romana que ordena el códice, llama la atención que esta representa el sistema vigesimal del francés actual (y de otras lenguas) a partir del número ochenta. De ese modo, el folio 80 está numerado 
como iiii xx (quatre-vingt); el 81 como iiii xxi y así sucesivamente. Igualmente, el folio 180 aparece numerado como $c$ iiii $x x$; el 181 como $c$ iiii $x x i$, etc.

Además de estos 271 folios que presentan numeración romana, el códice contiene otros dos sin ella: el primero y el último, los cuales presentan contenidos no homiléticos, sino de presentación y de conclusión. Estos dos folios se corresponden con el 1 y el 275 de la numeración establecida en el siglo XIX.

El folio 1 contiene, en el recto, una gran iluminación con la imagen de un copista y, en el verso, un caligrama que ocupa toda la página, con motivos laudatorios a Jesucristo sobre diversos episodios evangélicos; por su parte, en el recto del último folio (273r) se sitúa una cronología bíblica y, respectivamente, en el verso, un sermón inédito, sin el nombre de su autor.

\section{La iluminación del pantocrátor}

La iluminación del pantocrátor es más importante de todo el códice. Está situada en el folio 2 de la encuadernación actual, pero, como se dijo antes, correspondía al primer folio del manuscrito en su encuadernación original.

En ella se representa una escena, delimitada por la mandorla o almendra mística, formada por tres bandas: una negra, exterior, muy delgada; otra central, ancha y de color rojo; una interior de color pergamino decorada con mandorlinas en su interior. Por su parte, el fondo de la mandorla está dividido en cuatro bandas de tres colores, siguiendo el orden de arriba abajo: azul para cabeza de Cristo; verde para tronco; rojo para el centro de la mandorla; y verde nuevamente desde las rodillas de Cristo hasta los pies. La parte central de la mandorla, que abarca únicamente a Cristo, es de color pergamino y está delimitada por dos bandas que contienen las rogativas de san Andrés y de santa Maxelendis.

En la escena aparecen representados cuatro personajes: Jesucristo en toda su Majestad, dando la bendición con la mano derecha y sosteniendo un libro en la izquierda que contiene un mensaje dirigido a los dos personajes que 
lo flanquean a izquierda y a derecha: el apóstol san Andrés y santa Maxelendis. Completa la escena un fraile que, fuera de la mandorla, está postrado en actitud suplicante, sujetando los pies de Cristo y con el rostro vuelto a san Andrés, en señal de rogativa.

La iluminación de los personajes presenta las características propias de la pintura románica: líneas marcadas en los pliegues de las vestiduras, peinado simétrico, hieratismo. En el caso del Cristo, cejas arqueadas, mirada penetrante.

Para entender la escena representada, se debe comenzar, precisamente, por el fraile, el cual ha dejado escrito su nombre en un recuadro que sirve de escabel a los pies de Cristo (Figura 2). El nombre escrito que aparece es $F \hat{r}$ Raineru ${ }^{C}$; es decir: Frater Rainerus (el hermano Rainiero):

Figura 2: Detalle del folio 2-recto del Ms528 de Cambrai: Frater Rainerus

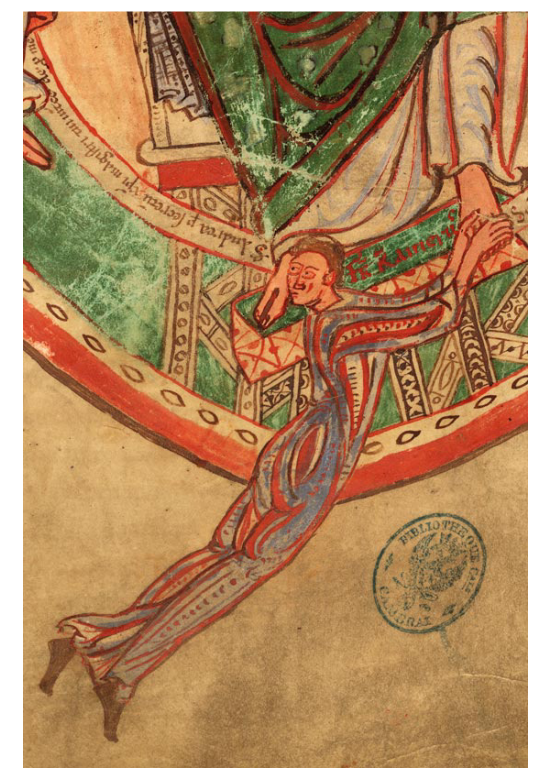

Fuente: Ministère de la Culture - Enluminures.

El catálogo virtual de las iluminaciones del manuscrito, elaborado por el Ministère de la Culture de Francia, a través del Institut de Recherche et d'Histoire des Textes - IRHT y del Centre National de la Recherche Scientifique - CRNS, identifica a Rainerus como copista del códice (Rainerus, 
copiste, écrivant), pues su imagen con el mismo rostro, peinado y ropajes también aparece en el folio anterior (1r), sentado en el scriptorium frente al atril y transcribiendo el Salmo I (Figura 3):

Figura 3: Fólio 1r do Liber sancti Andreae de castello (f001).

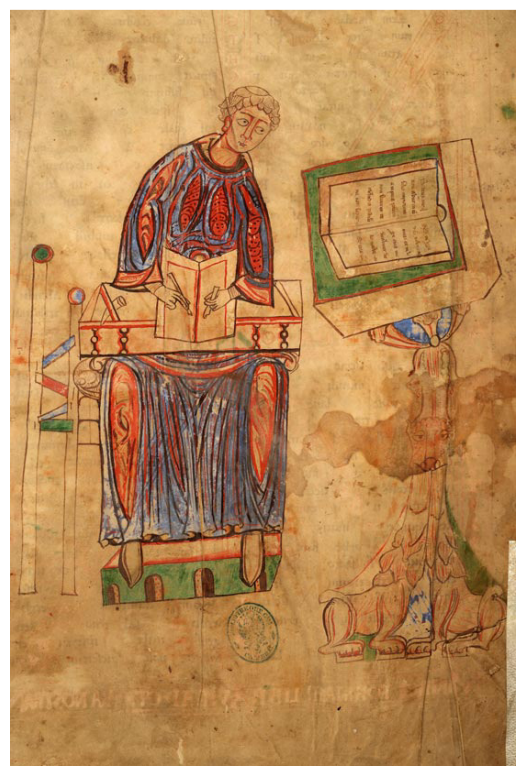

Fuente: Ministère de la Culture - Enluminures.

La aparición del autor en una iluminación que da inicio al códice es un recurso decorativo bastante frecuente en la confección de manuscritos medievales. Según Weitzmann (1977: 10), la modalidad de retrato de autor es la forma más antigua de iluminación en Occidente; por su parte, Sánchez Prieto (2015: 1) afirma que, en Codicología, el retrato de autor, junto a los laberintos, página tapiz, inicial decorada, representatio, praesentatio, propietario del libro y miniatura narrativa o inicial historiada, conforman las diferentes tipologías de decoración inicial de un códice.

Tras indagar en fuentes históricas de la región de Cambrai, se han podido hallar cuatro documentos relacionados con la época de elaboración del manuscrito que citan hasta cinco clérigos con el nombre de Rainerus, asociados a otros monasterios de la región en un radio no superior a 79 km de distancia de 
Cambrai, aunque no hay en los documentos informaciones explícitas que demuestren que alguno de los cinco sea el copista de Saint-André du Cateau.

El primero de ellos se refiere al año 1168, sobre una cesión de derechos de explotación de un bosque cercano, citando a un tal Rainiero, clérigo de Méricourt-l'Abbé, una población situada a 67 km de Cambrai:

Puesto que habiendo sido cedido igualmente por Rainiero, clérigo de Méricourt-l'Abbé, Egidio y Simón, los cuales tenían usufructo en el mismo bosque, estos lo concedieron y perdieron cualquier derecho sobre él (BEAUVILLÉ, 1860: 6, traducción propia).

El siguiente testimonio se refiere a una donación de grano en 1169 a la abadía de Fonsomme, a 36 quilómetros de Cambrai. En este caso, se cita a Rainiero, abad de Chauny, un monasterio a 79 km de la misma ciudad:

Refrendamos la presente página con la firma de los testigos al pie de la misma: sello del maestro abad Odón; sello del abad Pedro de Humblieres; sello de Rainiero, abad de Chauny [...]. Tramitado en el año de la encarnación del Señor de 1169 (BEAUVILLÉ, 1867: 11, traducción propia).

Después se tiene noticia de una donación de madera a la abadía de Vermandois en 1186. Esta abadía está situada a una distancia de $32 \mathrm{~km}$ de Cambrai. La cita se refiere a Rainiero de Moi (actual Moÿ-de-l'Aisne, a 65 km de Cambrai) y a Rainiero de Harbeis (actual Harbonnières, a 64 km):

Han firmado abajo, además, como testigos, el hermano Rainiero de Moi, el hermano Rainiero de Harbeis. Esta confirmación fue tramitada en Bapaume, en el año del Señor de 1186, en el mes de diciembre (BEAUVILLÉ, 1860: 50, traducción propia).

Además, está registrado entre 1189 y 1191 en el Annuaire du Conseil Héraldique de France (1895) un cierto "Rainier de Moy, chevalier de Saint Jean de Jérusalem, Frater Rainerus de Moi” (POLI, 1895: 268). 
Resulta pertinente recordar que la Abbaye du Saint-Sépulcre de Cambrai tuvo origen en la infausta ${ }^{2}$ peregrinación del obispo Lietberto a Tierra Santa, como se explicó antes en la cita textual de Sección 1, cuando se aludió a la fecha de construcción de la abadía en 1064 por iniciativa de este prelado.

Se puede leer un texto describiendo una línea curva que parte de Rainerus en dirección a san Andrés, con el siguiente ruego: "S Andrea $\rho$ fecretũ Xpĩ magiftri tui int̃cede p me"; es decir, Sancte Andrea: per secretum Christi, Magistri tui, intercede pro me (San Andrés: por el misterio de Cristo, tu Maestro, intercede por mí - Traducción propia).

La dirección de la mirada rogatoria de Rainerus nos conduce al destinatario de la misma, que está identificado con la siguiente inscripción: "Sc̃s ANDREAS Apłs"; o sea: sanctus Andreas Apostolus (san Andrés Apóstol), el patrono del monasterio de Sancti Andreae de castello (Saint André du Cateau). La causa del patronazgo de san Andrés se debe al fundador del monasterio, el obispo de Cambrai Gerard I (975 - 1051).

En la iluminación, el apóstol señala con el brazo extendido hacia abajo, fijando el dedo índice y la mirada en Rainerus, mientras que con el brazo derecho intenta llamar la atención o intermediar a favor del monje ante Cristo. La intercesión se describe en el mensaje que circunda la parte izquierda del torso de Cristo, describiendo una línea curva ascendente hasta llegar a su cabeza. Dice el mensaje: "Obfecro Dñe fac mifericordiã cum seruo tuo R.”; es decir: Obsecro, Domine, fac misericordiam cum servo tuo $R$. (Te ruego, Señor, ten misericordia de tu siervo R. - Traducción propia). La R. se refiere, naturamente, a Rainerus.

\footnotetext{
${ }^{2}$ Lietberto y su comitiva partió por tierra hacia el sureste, atravesando territorios del Sacro Imperio Romano Germánico hasta llegar a la región de Dalmacia y de allí, siguiendo la Costa Adriática hacia el sur, llegó hasta Corinto. La siguiente etapa lo llevó por mar hasta Laodicea, en el litoral de Asia Menor, donde fueron hostigados por los turcos, obligándoles a huir por tierra. Sufrieron penurias y enfermedades por el camino, lo que les obligó a desistir en el empeño de alcanzar los Santos Lugares y, de ese modo, emprendieron el camino de vuelta: primero hacia la costa y luego a través del mar hasta Chipre. En medio de tantas dificultades, el obispo pide la intercesión de la Virgen y de san Andrés, prometiéndoles un templo si les hacía retornar a Cambrai sanos y salvos, lo cual finalmente sucede. Todo el relato de la frustrada peregrinación se puede leer en tres fuentes de la época: en el Chronicon Andreas castri Cameracesii, en Vita sancti Lietberti y en Gesta Lietberti episcopi, dentro de los Monumenta Germaniae Historica (ver Bibliografía).
} 
El monje expresa otra rogativa que se puede leer a su derecha, siguiendo una línea curva ascendente, iniciándose en el pie derecho de Cristo y dirigida a santa Maxelendis: "Sub tuã protectionẽ confugio beata uirgo maxelendis"; o sea: Sub tuam protectionem confugio, beata virgo Maxelendis (Me refugio bajo tu protección, bienaventurada virgen Maxelendis - traducción propia).

Al lado derecho de la imagen, dentro de la mandorla e identificada mediante la inscripción "Sc̃a maxeLeNdis" aparece la figura de santa Maxelendis, una santa mártir oriunda de la región de Cambrai cuyas reliquias fueron confiadas en 1025, según Bertrand y Mériaux (2006: 3), a la Abbaye Saint-Andre du Cateau. La legendaria historia de su martirio está recogida en cinco fuentes latinas medievales:

En orden cronológico, el primero que la cita es Beda el Venerable (672 - 735) en su Martyrologium: "En el territorio de Cambrai, en un poblado que se llama Caudry, fue el martirio de santa Maxelendis, virgen y mártir" (BEDA, 1850, p1103, traducción propia).

Después, encontramos una referencia en Sigeberto de Gembloux (1030 - 1112), escritor, cronista y monje benedictino belga. En su obra Chronica, en relación a los hechos importantes acaecidos en el año 670, se lee:

En el territorio de Cambrai, la virgen de Dios Maxelendis, huyendo de su enamorado Harduino, fue martirizada por este, por defender su voto de castidad (SIGEBERTO DE GEMBLOUX, 1854: 127, traducción propia).

La referencia más prolífica sobre esta mártir la encontramos en Gesta Pontificorum Cameracensium. Al comienzo de la obra se narran los orígenes de la ciudad y las historias legendarias de sus mártires:

En el tiempo de este santo hombre, la virgen de Dios Maxelendis es martirizada y su asesino se queda ciego:

Casi más o menos en esa época, cierta virgen en el territorio de Cambrai, nacida de noble cuna es acosada por cierto joven no de origen innoble, pero sí de ánimo innoble, para que se acostase con ella con la promesa de matrimonio. La cual, como no había podido ser persuadida ni con oro, ni con gemas ni con ningún otro tipo de adornos para que cediese en su propósito de virginidad y se uniera a un hombre poderoso según la costumbre de las mujeres nobles, y tampoco quería 
que sus padres oyesen sobre una decisión de este tipo, siendo desesperado el amor del joven y siendo aprovechada la oportunidad en la que pudiese atacar a la muchacha sola en la casa de sus padres, llevando consigo una banda de amigos malvados, rodearon toda la casa e, irrumpiendo en esta, la revisaron de arriba abajo y, finalmente, contemplando con curiosidad un pequeño escondrijo en el que la doncella se había escondido, la encontraron y la arrastraron violentamente hasta el lugar donde ahora está construida la basílica en honor de todos los santos, principalmente y sobre todo de san Vedasto y ciertamente también de veneración de esta santa doncella. Entonces, el funesto carnicero, apretando los dientes la mata golpeándola con la espada desenvainada. El cual, tan pronto como vio la sangre de la doncella, se quedó ciego por el juicio justo de Dios. Y fue sepultada en la basílica de los santos apóstoles Pedro y Pablo y del confesor san Sulpicio que fue construida en la población que se llama Pomeriolas.

En relación a lo cual, el santo obispo llevó el cuerpo de la doncella de vuelta al lugar del crimen y Harduino recobró la vista:

Habiendo transcurrido, sin embargo, tres años desde su pasión, cierta matrona noble llamada Amaltrudis, es advertida por una voz divina de que debía dirigirse al santo obispo de Cambrai, Vindiciano, para que este con su clero y la plebe vecina llevara el sagrado cuerpo de vuelta al lugar del crimen con suma veneración, porque el Señor había dispuesto destacar aquel lugar con señales y milagros. A esto, el santo obispo, en cuanto oyó las palabras de la que se lo contaba, se dispuso a cumplirlas. Después, Harduino, el que la había matado (es preciso decir que de manera miserable y con ignominia), vivía todavía en las tinieblas de su ceguera. El cual, cuando oyó que el sagrado cuerpo de la doncella fue llevado de vuelta al lugar de infausto recuerdo, maldiciéndose y reconociendo su pecado, ordena que lo lleven al encuentro del cuerpo y se postró ante el féretro, y en el mismo momento en que los portantes del sagrado cuerpo se llegaron a él, recobró la vista. Y corriendo hacia el obispo le contó cuanto había sucedido. Así pues, por motivo del presente milagro y habiéndoselo prometido al pueblo, el obispo dio sepultura al sagrado cuerpo en la iglesia citada anteriormente (ANÓNIMO, 1853: 40-1, traducción propia).

Al igual que la imagen de san Andrés, la figura de santa Maxelendis está completada por el mensaje de intercesión que esta le transmite al Cristo sedente: "Dnẽ ne auertaf faciẽ tuã a puero tuo R.”; esto es: Domine, ne avertas faciem tuam a puero tuo $R$. (Señor, no apartes tu rostro de tu hijo R. - 
traducción propia). Nuevamente, la R. se refiere al hermano Rainerus. El mensaje dibuja una línea curva ascendente hasta llegar a la cabeza de Cristo.

Las rogativas de los dos santos, pues, llegan a los oídos de Cristo, la figura central, sedente, en toda su majestad, con el rostro hierático y dando la bendición con su diestra, con dos dedos a la manera griega, al tiempo que sostiene un libro abierto en la izquierda con la respuesta a las rogativas de san Andrés y de santa Maxelendis, en color rojo (Tabla 1):

Tabla 1: Recreación del libro abierto en el folio 2-recto del Ms528 de Cambrai

\begin{tabular}{|c|c|c|}
\hline Pro eo & & Nc̃ \\
\hline quod & & iam \\
\hline rogal & & fiat \\
\hline tis me & & illi \\
\hline exau & & ficut \\
\hline diui & & petil \\
\hline uos.. & & tis.. \\
\hline & O & \\
\hline
\end{tabular}

Fuente: El autor.

Transcrito a caractertes más legibles y ordenado de izquierda a derecha, el texto viene a ser: Pro eo quod rogastis me exaudivi vos. Nunc iam fiat illi sicut petistis; es decir: "En favor de eso que me rogasteis, os escuché. Ahora, ya, cúmplasele según pedisteis" (traducción propia). La respuesta de Cristo a las rogativas en favor del monje Rainerus es, pues, favorable y se destaca en un rojo más intenso que el del resto de palabras el adverbio iam (ya, ahora, en este momento), para dar mayor énfasis.

\section{La poesía en latín}


En el margen derecho del folio, hacia el centro, hay una inscripción manual posterior en letra cursiva moderna con el texto de un dístico recogido en la Antología Latina, catalogado como $785^{\mathrm{a}}$, del que se dice en la misma que fue atribuido por la tradición a Agustín de Hipona, en virtud de un manuscrito Riccardianus 931, del siglo XVI, folio 15.

El dístico en cuestión dice lo siguiente: "Dulcia non meruit qui non gustavit amara et qui non studuit sunt illi gaudia rara", "No mereció lo dulce el que no probó lo amargo y escasas satisfacciones tiene el que no se esforzó" (traducción propia).

En otras antologías posteriores de poesía latina se titula a este breve poema como Dulcia post amara, "Lo dulce tras lo amargo" (PÉREZ DE ANTÁ, 1683: 6) o Gaudium dulce, "Dulce gozo" (GIBBS, 2012: 168).

En cualquier caso, este poema no forma parte de la imagen ni fue escrito en época medieval. Se trata apenas de un ejercicio de libre albedrío de alguien que tuvo acceso al códice y tuvo además la ocurrencia de escribir sobre este folio $2 \mathrm{r}$ un poema de su agrado.

\section{La inscripción del Magister del siglo XIX y la datación del manuscrito}

En la parte inferior derecha del folio, puede verse una anotación hecha por un bibliotecario o investigador del siglo XIX, quien deja su nombre, el año y la declaración de autoría de revisión o catalogación y su firma. Se ve también una sencilla operación matemática de resta que se refiere a los años transcurridos (712) entre dos momentos específicos no descritos (1827 y 1115):

"Magister Johannes Folksperg in 93. Par moy. (Firma). $1827-1115=$ 0712”.

El apellido Folksperg, de origen germánico, aparece en el repositorio digital del Centre des Recherches sur l'Histoire des Families - CRHF como una genealogía de la región de Alsacia y Lorena, una región a unos 400 quilómetros de Cambrai, de influencia alemana. 
El año 93 que se cita en la anotación del Magister Johannes no viene acompañado del siglo al que pertenece, pero considerando que el monasterio del Santo Sepulcro, lugar en el que estaba depositado el manuscrito, fue clausurado en 1791 por motivo de la Revolución Francesa, cabe pensar que se trate de una revisión, catalogación, incluso reencuadernación, relacionadas con su traslado a una nueva sede en esa misma época, por lo que parece lógico deducir que el año que se cita sea el de 1793.

La operación matemática 1827 - 1115 presenta una caligrafía diferente a la anterior, no fue hecha por el Magister, por lo que se debe pensar que hubo una nueva revisión del manuscrito posterior a 1793. Ahora bien, no se ofrecen mayores informaciones sobre las dos fechas de referencia.

El último elemento que forma parte del folio, en su parte inferior, es el sello de la Biblioteca de Cambrai, último lugar de reposo del Ms528.

\section{Conclusión}

El folio 2-recto del manuscrito Ms528 de Cambrai aporta informaciones relevantes sobre la historia del códice, las diferentes sedes donde estuvo depositado y las personas que intervinieron en él. Además, la iluminación que contiene es, en sí misma, ejemplo de decoración inicial de un códice que da noticias sobre el autor, el monje benedictino Rainerus, que fue la persona más antigua en manipular el códice de las que se tiene noticia, en su faceta como copista o como iluminador.

El manuscrito estuvo depositado, a lo largo de su historia en, al menos, tres lugares diferentes: 1) la Abbaye Saint-Andre du Cateau, en la región de Le Cateau-Cambrésis, lugar donde fue elaborado/copiado en el siglo XII; 2) la Abbaye $d u$ Saint-Sépulcre de Cambrai, donde fue nuevamente revisado/catalogado y encuadernado. Allí estuvo hasta la época de la Revolución Francesa, cuando fue clausurado el monasterio; 3) la Bibliotèque Municipale de Cambrai, lugar donde está depositado actualmente y que ha digitalizado su contenido para su consulta pública en la red. 
Hay cuatro fechas de referencia que aporta el documento: la primera por orden cronológico es 1115, anotada por alguien que consultó el manuscrito en el siglo XIX; la segunda es 1170, según la cronología papal añadida en el último folio, que identifica esa fecha con el papa Alejandro III; la tercera es 1793, cuando el códice fue revisado por un tal Magister Johannes Folksperg; la cuarta y última es 1827 , que alguien relacionó con 1115 para conocer la diferencia de tiempo entre ambas (712).

Existe una numeración antigua del manuscrito en números romanos, establecida en la época en que fue elaborado, que no considera el primero ni el último folio y otra moderna en números arábigos establecida por un catalogador o bibliotecario posterior, asumida después también por la Bibliothèque Municipale de Cambrai, que sí los considera. Esto nos lleva a pensar que, originalmente, el primer folio y el último no formaban parte del códice y fueron añadidos con posterioridad en el momento de su reencuadernación.

De ese modo, el folio del pantocrátor era el $i$ de la numeración original y se corresponde con el 2 de la numeración actual; el ii con el 3 y así sucesivamente hasta el $c$, que lo hace con el 101. Al faltar, como se dijo antes, la numeración $c i$ en el original, coinciden a partir de ahí las dos numeraciones hasta el folio cxxxix, donde se produce el otro salto: el folio cii se corresponde con el 102, etc., pero el cxli se corresponde con el 140, el cxlii con el 141, etc., y así hasta el final del códice, donde cclxxiii es 274 y el último folio, sin numerar, es el 275.

Sin embargo, a pesar de los varios saltos en la numeración de los folios, tanto en la antigua como en la moderna posterior, el texto no está interrumpido entre los folios anterior y posterior a los saltos, no hay lagunas en el texto del manuscrito. Es decir: según la lectura de los textos, no falta ningún folio en el manuscrito.

Según esto, resulta necesario que la Bibliothèque Municipale de Cambrai haga una nueva numeración de los folios, que debe ir desde el 001 hasta el 273, que es el número exacto de folios que contiene el códice, después del análisis realizado. 
En cuanto a la iluminación en sí, esta representa una escena rogativa del monje Rainiero a dos santos intercesores, la cual se desarrolla en el sentido de las agujas del reloj, comenzando por el propio interesado, situado en la parte baja de la imagen, que se dirige a su abogado defensor san Andrés, continúa hacia el lado opuesto donde se sitúa santa Maxelendis, la cual también intercede por Rainiero. Finalmente, Cristo da venia a la solicitud de los dos santos, refrendándolo por escrito en un libro abierto.

Sin embargo, el folio no aporta informaciones sobre cuál es el motivo por el que Rainiero pide la intercesión de los santos.

Se pudo comprobar también que el folio en cuestión fue objeto de cierto vandalismo por alguien que tuvo la ocurrencia de escribir una poesía en latín al lado del pantocrátor.

\section{Referências}

ANÓNIMO. Chronicon sancti Andreae Cameracesii. París. J. P. Migné, 1853. Disponible en: <<http://www.documentacatholicaomnia.eu/02m/10951076,_Anonymus,_S_Andreae_Castri_Cameracensi_Chronicon,_MLT.pdf $>>$. Acceso en: 11 de diciembre de 2019.

ANÓNIMO. Gesta Pontificorum Cameracensium. Paris. J. P. Migné, 1853. Disponible en: $\quad<$ http://www.documentacatholicaomnia.eu/02m/10911174,_Auctor_Incertus,_Gesta_Pontificorum_Cameracensium,_MLT.pdf $>>$. Acceso en 26 de mayo de 2019.

ANTOLOGÍA LATINA. Repertorio de poemas extraído de códices y libros impresos. Madrid. Editorial Gredos, 2011.

BEAUVILLÉ, V. de. Recueil de documents inédits concernant La Picardie. París: L'Imprimerie Impériale, 1860. Disponible en: < https://numelyo.bmlyon.fr/f_view/BML:BML_00GOO0100137001101342397?pid=BML:BML_0 0GOO0100137001101342397\&pg titre $=>$. Acceso en 29 de mayo de 2019.

BEAUVILLÉ, V. de. Recueil de documents inédits concernant La Picardie. Deuxième parte. París: L'Imprimerie Impériale, 1867. Disponible en: < https://reader.digitalesammlungen.de/de/fs1/object/display/bsb10358247_00630.html>.

BEDA. Martyrologium. París. J. P. Migné, 1850. Disponible en: < http://www.documentacatholicaomnia.eu/02m/0627- 
0735,_Beda_Venerabilis,_Martyrologium_[Editio_Coloniensis],_MLT.pdf>. Acceso en 25 de mayo de 2019.

BEDA. Opera Paraenetica sive omnius eius operum pars tertia. París. J. P. Migné, $1850 . \quad$ Disponible en: $<$ http://www.documentacatholicaomnia.eu/02m/0627-

0735,_Beda_Venerabilis,_Homiliae,_MLT.pdf $>$. Acceso en 03 de abril de 2019.

BERTRAND, P.; MÉRIAUX, C. Cambrai-Magdebourg: les reliques des saints et l'intégration de la Lotharingie dans le royaume de Germanie au milieu du Xe siècle. En: Médiévales. $\mathrm{N}^{\circ}$ 51, Automne 2006: L’Occident sur ses marges $\left(\mathrm{VI}^{\mathrm{e}}\right.$ - $\mathrm{VI}^{\mathrm{e}}$ siècles), p.85-96. Disponible en: $<$ https://journals.openedition.org/medievales/1514 >. Acceso en 29 de mayo de 2019.

BEthmanN, L. K. Chronicon sancti Andreae castri Cameracesii. París. J. P. Migne, $1853 . \quad$ Disponible en: < http://www.documentacatholicaomnia.eu/02m/10951076,_Anonymus,_S_Andreae_Castri_Cameracensi_Chronicon,_MLT.pdf > Acceso el 22 de mayo de 2019.

BUECHELER, F.; RIESE, A. Anthologia Latina sive Poesis Latinae Supplementum. Leipzig. Teubner, 1894. Disponible en: $<$ https://warburg.sas.ac.uk/pdf/eph30b2440713.pdf $>$. Acceso en: 28 de agosto de 2018.

BVMM BIBLIOTHÈQUE VIRTUELLE DES MANUSCRITS MÉDIÉVAUX. 7. Cambrai, Bibliothèque municipale 0528 (0487), f001. Disponível em: $<$ https://bvmm.irht.cnrs.fr/consult/consult.php?VUE_ID=1712693>. Acesso em 08 de dezembro de 2019.

BVMM BIBLIOTHÈQUE VIRTUELLE DES MANUSCRITS MÉDIÉVAUX. 7. Cambrai, Bibliothèque municipale 0528 (0487), f002. Disponible en: < https://bvmm.irht.cnrs.fr/consult/consult.php?mode=ecran\&reproductionId=103 $12 \&$ VUE_ID $=1294698 \&$ panier $=$ false \&carouselThere $=$ false \&nbVignettes $=4 \times 3$

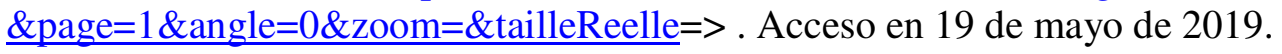

BVMM BIBLIOTHÈQUE VIRTUELLE DES MANUSCRITS MÉDIÉVAUX. Cambrai, Bibliothèque municipale 0528 (0487), f002 - Vue 4. Disponible en: < https://bvmm.irht.cnrs.fr/consult/consult.php?mode=ecran\&reproductionId=103 $12 \& V U E \_I D=1294696 \&$ panier=false \&carouselThere=false \&nbVignettes $=4 \times 5$ \&page $=1 \&$ angle $=0 \&$ zoom $=\&$ tailleReelle $=>$. Acceso en 25 de mayo de 2019.

CARPENTIER, J. B. Histoire de Cambrai et du Cambrésis. Leiden. Chez L'Autheur, 1664. Disponible en: < https://books.google.com.br/books?id=pSgAAAAcAAJ\&pg=RA1- 
PA100\&hl=es\&source $=g b s \_$selected_pages $\&$ cad $=3 \# \mathrm{v}=$ onepage $\& \mathrm{q} \& \mathrm{f}=$ false $>$.

Acceso en 19 de mayo de 2019.

GIBBS, L. Brevissima: 1001 Tiny Latin Poems. Morrisville. Gibbs, 2012. Disponible en: <http://brevissima.bestlatin.net/brevissima-v1.pdf $>$. Acceso en 30 de agosto de 2018.

GODOI, P. W. O copista de Cambrai: Rainerus e a representação do artista na Idade Média. In: XIX SEMANA DE HISTÓRIA VII FORUM DE PÓSGRADUAÇÃO EM HISTÓRIA E II FORUM DE LICENCIATURA EM HISTÓRIA REALIZADA - O PROFISSIONAL DE HISTÓRIA E SEUS DESAFIOS. TEMÁTICA DE ENSINO E PESQUISA, 1, 2015, Anais [...], Maringá (UEM), 2015, p. 587-598.

JERÓNIMO DE ESTRIDÓN. Operum mantissa continens scripta supposititia. París, J. P. Migné, 1846. Disponible en: $<$ http://www.documentacatholicaomnia.eu/02m/0347-

0420, Hieronymus, Epistolae, MLT.pdf>. Acceso en 24 de mayo de 2019.

MARTINIÈRE, A.-A. B. de la. Le grand dictionnaire geographique, historique e critique. París. Boudet, 1768. Disponible en: $<<$ https://books.google.fr/books?id=4MTLsBwu3ygC\&pg=PA218\&dq=Abbay e+du+Cateau+Saint-

Andr\%C3\%A9\&hl=fr\&sa=X\&ei=cvNsUaiJKZSe7AaHj4HABg\&ved=0CEsQ6 AEwBA\#v=onepage \&q=Abbaye $\% 20 \mathrm{du} \% 20$ Cateau\%20SaintAndr\%C3\%A9\&f=false $>>$. Acceso en 27 de mayo de 2019.

MINISTÈRE DE LA CULTURE. Enluminures. Cambrai - BM - Ms. 528 f001. Disponível em: $<$ http://www.enluminures.culture.fr/documentation/enlumine/fr/BM/cambrai_0 23-01.htm>. Acesso em 08 de dezembro de 2019.

MINISTÈRE DE LA CULTURE. Enluminures. Cambrai - BM - Ms. 528 f002. Disponible en: < http://www.enluminures.culture.fr/documentation/enlumine/fr/BM/cambrai 02 3-01.htm>. Acceso en 25 de mayo de 2019.

MONUMENTA GERMANIAE HISTORICA. Scriptorum Tomus XII. Hannover. G. H. Pertz, 1856. Disponible en: $<$ http://www.documentacatholicaomnia.eu/20vs/201_MGH/19251933,_MGH,_1_Scriptores._SS_12._Historiae_Aevi_Salici,_LT.pdf>. Acceso en 11 de diciembre de 2019.

PÉREZ DE ANTÁ Y LOYOLA, J. Hortulus Carminum Selectorum. Bruselas. Jacobi van de Velde, 1683. Disponible en: $<$ https://books.googleusercontent.com/books/content?req=AKW5QaelY7Q1q2 RtXQGL4ltjPNH89fu2kLM1amKrw_OILUrkySYTw14UW38sg53PhqALnpg 
FoCQKn6kZT82qIWv3wWrUTtEg676Ml47CQRrl0ectHJySSjDDBGpTsCeV ZAPxoWBQZi5Iv-pweVfkn4Rimq59q9vdkTY7JeQZTIDeGkLsAr_8h_AWNEXIFAS01R_18-XoRdYKsQPe2dDHiCk7R8GzmBoTOvqKibUYn_RNEI079LlgCY8H_o0csTxTihgvrat 0JTtl_rvQeUf 8CBT9wah9gcWBtxGv6-VNe5DmYreet8>. Acceso en 28 de agosto de 2018.

RODULFUS. Vita Lietberti episcopi Cameracensis auctore Rodulfo monacho S. Sepulchri Cameracensis. Leipzig, 1926. Hofmeister Ed.

POLI, O. de. Croisés de France (1095 - 1396) p. 180 - 303. En: Annuaire du Conseil Héraldique de France. París: Bibliotèque Nationale de France, 1895. Disponible en: $<$ http://gallica.bnf.fr/ark:/12148/bpt6k368901/f1.image.langFR> . Acceso en: 29 de mayo de 2019.

SÁNCHEZ PRIETO, A. B. Decoración del códice. 4. La decoración inicial. En: Academia.es. 2015.2 Disponible en: < https://www.academia.edu/28844607/La decoraci\%C3\%B3n del c\%C3\%B3di ce._2._La_escritura_convertida_en_elemento_decorativo $>$. Acceso en 26 de mayo de 2019.

SIGEBERTO DE GEMBLOUX. Chronica. Paris. J. P. Migne, 1854. Disponible en: < http://www.documentacatholicaomnia.eu/02m/10301112,_Sigebertus_Gemblacensis,_Chronica,_MLT.pdf $>$. Acceso en 25 de mayo de 2019.

WEITZMANN, K. Late Antique \& Early Christian Book Illumination. Nueva York. George Braziller, 1977.

Anexo

Contenido íntegro del manuscrito Ms528 de Cambrai por autores/ obras:

Alcuino de York:

- Vita sancti Vedasti. Adhortatio ad imitandas virtutes santi Vedasti in actis descriptas.

Ambrosio Autperto:

- Sermo de lectione evangelica.

Ambrosio de Milán:

- De virginibus. Liber Primus, Caput II - III.

- De virginibus. Liber Primus, Caput V-VIII.

- Exhortatio virginitatis, Caput IV - V.

- Expositio Evangelii secundum Lucam, Liber Secundus, Caput II, vs. xxv $-x l i$.

- Expositio Evangelii secundum Lucam, Liber Sextus, Caput IX. 
- Expositio Evangelii secundum Lucam, Liber Septimus, Cap. X.

Autor desconocido:

- Sermo de nativitate sanctae Mariae.

- Sermo de sancta Trinitate.

- Sermo de sepulchro Domini.

- Sermo de vinculis sancti Petri Apostoli.

- Sermo post tempus stellae.

Augustín de Hipona:

- De civitate Dei. Liber XXII. Caput VIII. vs. $x$-xxii.

- De sermone Domini in monte, Liber Primus, Cap. IX-X.

- In Psalmum LXIII enarratio.

- In Psalmum LXIV enarratio. Sermo ad plebem, III - XVIII.

- In Psalmum LXXXIII enarratio, V-XVII.

- In Psalmum LXXXVI enarratio, II - IX.

- In Psalmum CXXI enarratio. II - V; X-XIV.

- In Psalmum CLXVII enarratio. VII - IX; XIII; XX - XXVI.

- Sermo XIX. Item de dedicatione Ecclesiae II.

- Sermo XXXI de Scripturis. De verbis Psalmi CXXV.

- Sermo LXXII. De verbis Evangelii Matthaei, Cap. XIV, xxiii-xxxii.

- Sermo XCIII. De verbis Evangelii Matthaei.

- Sermo CLXXV de tempore. De letania.

- Sermo CXC. In Cathedra sancti Petri, I.

- Sermo CXCI. In Cathedra sancti Petri, II.

- Sermo CCLXXXVII. In natali sancti Iohannis Baptistae.

- Sermo CCVIII. In festo Assuptionis beatae Mariae.

- Sermo CCXX. In natale sanctorum Innocentium.

- Tractatus X in Iohannis Evangelium.

- Tractatus XII in Iohannis Evangelium.

- Tractatus XXVI in Iohannis Evangelium.

- Tractatus XLV in Iohannis Evangelium.

- Tractatus LI in Iohannis Evangelium.

- Tractatus LII in Iohannis Evangelium.

- Tractatus LXVII in Iohannis Evangelium.

- Tractatus LXVIII in Iohannis Evangelium.

- Tractatus LXIX in Iohannis Evangelium.

- Tractatus LXX in Iohannis Evangelium.

- Tractatus LXXI in Iohannis Evangelium.

- Tractatus LXXX in Iohannis Evangelium. 
- Tractatus LXXXI in Iohannis Evangelium.

- Tractatus LXXXII in Iohannis Evangelium.

- Tractatus LXXXIII in Iohannis Evangelium.

- Tractatus LXXXIV in Iohannis Evangelium.

- Tractatus LXXXV in Iohannis Evangelium.

- Tractatus LXXXVI in Iohannis Evangelium.

- Tractatus LXXXVII in Iohannis Evangelium.

- Tractatus LXXXVIII in Iohannis Evangelium.

- Tractatus LXXXIX in Iohannis Evangelium.

- Tractatus XC in Iohannis Evangelium.

- Tractatus XCI in Iohannis Evangelium.

- Tractatus CV in Iohannis Evangelium.

- Tractatus CVI in Iohannis Evangelium.

- Tractatus CVII in Iohannis Evangelium.

- Tractatus CXIX in Iohannis Evangelium.

- Tractatus CXXIII in Iohannis Evangelium.

Beda:

- In Evangelium sancti Lucae, Liber Secundus, Cap. IX.

- In Evangelium sancti Lucae, Liber Secundus, ante Caput V.

- In Evangelium sancti Lucae, Liber Secundus, Caput V.

- In Evangelium sancti Lucae, Liber Secundus. Caput XX.

- In Evangelium sancti Lucae, Liber Tertius, Cap. XI.

- In Evangelium sancti Lucae, Liber Tertius, Caput VIII.

- In Evangelium sancti Lucae, Liber Quartus, Invocatio.

- In Evangelium sancti Lucae, Liber Quartus, Caput XII.

- In Evangelium sancti Lucae, Liber Quintus, Cap. XIX.

- In Evangelium sancti Lucae, Liber Quintus, Caput XX.

- In Evangelium sancti Marci, Liber Primus, Cap. III.

- In Evangelium sancti Marci, Liber Secundus, Cap. VI.

Liber Primus Homiliarum (homiliae genuinae):

- Homilia VIII. In die natali sancti Iohannis Baptistae.

- Homilia IX. In die festo Innocentium.

- Homilia XV. In purificatione beatae Mariae.

Liber Secundus Homiliarum (homiliae genuinae):

- Homilia I. In vigilia Paschae.

- Homilia II. In vigilia Paschae.

- Homilia III. In feria sexta inter octavam Paschae. 
- Homilia IV. Infra octauas Paschae.

- Homilia V. In dominica secunda post octavas Paschae.

- Homilia VI. In tertia dominica post sanctum Pascha.

- Homilia VII. In quarta dominica post sanctum Pascha.

- Homilia VIII. In litania maiore.

- Homilia IX. In Ascensione Domini.

- Homilia X. In dominica post Ascensionem Domini.

- Homilia XI. In Vigilia Pentecostes.

- Homilia XII. In octava Pentecostes.

- Homilia XIII. In vigilia sancti Iohannis Baptistae.

- Homilia XIV. In nativitate sancti Iohannis Baptistae.

- Homilia XV. In vigilia beatorum apostolorum Petri et Pauli.

- Homilia XVI. In natale beatorum apostolorum Petri et Pauli.

- Homilia XVII. In natale sancti Benedicto Episcopo.

- Homilia XX. In decollatione sancti Iohannis Baptistae.

- Homilia XXI. In dedicatione Ecclesiae.

- Homilia XXII. In natali sancti Matthaei Apostoli.

- Homilia XXIII. In natali sancti Andreae Apostoli.

Liber Tertius Homiliarum (homiliae subdiditae):

- Homilia IV. In dominica quarta post Trinitatem.

- Homilia V. In dominica quinta post Trinitatem.

- Homilia VI. In dominica septima post Trinitatem.

- Homilia X. In dominica undecima post Trinitatem.

- Homilia XI. In dominica duodecima post Trinitatem.

- Homilia XII. In dominica decima tertia post Trinitatem.

- Homilia XIII. In dominica decima quarta post Trinitatem.

- Homilia XIV. In dominica decima quinta post Trinitatem.

- Homilia XV. In dominica decima sexta post Trinitatem.

- Homilia XVI. In dominica septima decima post Trinitatem.

- Homilia XXI. In septembris feria quarta quatuor temporum.

- Homilia LV. In natali divae Mariae Virginis.

- Homilia LVII. In die Assumptione Mariae.

- Homilia LX. In natali divi Bartholomaei Apostoli.

- Homilia LXV. In dedicatione Ecclesiae.

- Homilia LXVI. In dedicatione Ecclesiae.

- Homilia LXIX. In solemnitatem Omnium Sanctorum.

- Homilia LXX. In eadem solemnitate Omnium Sanctorum.

- Homilia LXXI. In eadem solemnitate Omnium Sanctorum. 
- Homilia LXXII. In die sancto unius apostoli.

- Homilia LXXIII. In festo Martyrum.

- Homilia LXXVII. In festo confessorum prima.

Biblia Sagrada:

- Lamentationes, IV, i-xxii.

- Liber Tertium Regum, VIII, xxii-l.

Eusebio de Cesarea:

- Historia Ecclesiastica. Liber III, Caput XXIII.

Fulgencio de Ruspe:

- Sermo I. De dispensatoribus Domini, Cap. I - VIII.

- Sermo I. De dispensatoribus Domini, Cap. IX - XIII.

- Sermo II. In natali sancti Stephani.

- Sermo III. De sancto Stephano Protomartyre et conversione sancti Pauli.

- Sermo IV. De Epiphania Domini et de Innocentibus.

- Sermo LVII. De santo Petro, ubi dicit Dominus: "Pasce oves meas".

- Sermo LVIII. In natali Apostolorum Petri et Pauli.

Gregorio I:

- Homilia III in Evangelia.

- Homilia V in Evangelia.

- Homilia IX in Evangelia.

- Homilia XI in Evangelia.

- Homilia XII in Evangelia.

- Homilia XIII in Evangelia.

- Homilia XIV in Evangelia.

- Homilia XVII in Evangelia.

- Homilia XXI In Evangelia.

- Homilia XXII in Evangelia.

- Homilia XXIII in Evangelia.

- Homilia XXIV in Evangelia.

- Homilia XXV in Evangelia.

- Homilia XXVI in Evangelia.

- Homilia XXVII in Evangelia.

- Homilia XXVIII in Evangelia.

- Homilia XXIX in Evangelia.

- Homilia XXX in Evangelia.

- Homilia XXXI in Evangelia. 
- Homilia XXXII in Evangelia.

- Homilia XXXIII in Evangelia.

- Homilia XXXIV in Evangelia.

- Homilia XXXV in Evangelia.

- Homilia XXXVI in Evangelia.

- Homilia XXXVII in Evangelia.

- Homilia XXXVIII in Evangelia.

- Homilia XXXIX in Evangelia.

- Homilia XL in Evangelia.

Jerónimo de Estridón:

- Commentaria in Matthaeum. Liber Primus, Cap. VII, vs. xv-xxi.

- Commentaria in Matthaeum. Liber Primus, Cap. X, vs. xxvi-xxxiii.

- Commentaria in Matthaeum. Liber Primus, Cap. X, vs. xxxiv-xlii.

- Commentaria in Matthaeum. Liber Secundus, Cap. XIII, vs. xxiv-xliii.

- Commentaria in Matthaeum. Liber Secundus, Cap. XIV, vs. xxii-xxx.

- Commentaria in Matthaeum. Liber Tertius, Cap. XVI, vs. xiii-xix

- Commentaria in Matthaeum. Liber Tertius, Cap. XVIII, vs. $i-x i$.

- Commentaria in Matthaeum. Liber Tertius, Cap. XVIII, vs. xxiii-xxxv.

- Commentaria in Matthaeum. Liber Tertius, Cap. XIX, vs. xxvii-xxx

- Commentaria in Matthaeum. Liber Tertius, Cap. XX, vs. xx-xxiii.

- Commentaria in Matthaeum. Liber Tertius, Cap. XXII, vs. xxxvi-xlii.

- Commentaria in Matthaeum. Liber Quartus, Cap. XXIII, vs. xxxiv-ix.

- Commentaria in Matthaeum. Liber Quartus, Cap. XXIV, vs. xxxvi-xlvii.

- Commentaria in Matthaeum. Liber Quartus, Cap. XXV, vs. $i$-xiii.

- De Assumptione beatae Mariae Virginis.

- De virginitate beatae Mariae, adversus Helvidium, Liber I.

- Epistula IX. Ad Paulam et Stochium. De Assumptione beatae Maria.

- Epistula XXIV, seu sermo de resurrectione Domini, Cap. VII - XIII.

- Epistula L. De nativitate sanctae Mariae, Cap. I.

- Epistula L. De nativitate sanctae Mariae, Cap. II - XI.

- Epistula CXX. Ad Hebidiam, de quaestionibus XII, Cap. IV-V.

- Epistula CXXI. Ad Algasiam, Cap. VI.

Hilario de Poitiers:

- Tractatus super Psalmos, Littera VIII, Heth.

Juan Crisóstomo:

- Homilia de David, ubi Goliath immanem hostem devicit.

- Homilia III. De laudibus sancti Pauli apostoli. 
- Homilia XXVIII.

- Homilia XXXIX. In natali Innocentium.

- Homilia XLII in Matthaeum.

Isidoro de Sevilla:

- De ortu et obitu patrum. Ex Novum Testamentum, Cap. LXXII.

Lorenzo de Novara:

- Homilia prima de poenitentia.

León I:

- Sermo LXXIII. De ascensione Domini I.

- Sermo LXXIV. De ascensione Domini II.

- Sermo LXXV. De Pentecoste I.

- Sermo LXXVI. De Pentecoste II.

- Sermo LXXVII. De Pentecoste III.

- Sermo LXXXII. In natale apostolorum Petri et Pauli.

- Sermo XCV sive homilia de gradibus ascensionibus ad beatitudinem.

Máximo de Turín:

- Homilia LIII. De poenitentia Petri et ostiaria ancilla.

- Homilia LVII. De eadem Paschae sollemnitate III.

- Homilia LVIII. De eadem Paschae sollemnitate IV.

- Homilia LX. De Ascensione Domini.

- Homilia LXII. De solemnitate sancta Pentecostes II.

- Homilia LXIV. In natali sancti Stephani Levitae et Protomartyris.

- Homilia LXV. In die nativitatis sancti Iohannis Baptistae I.

- Homilia LXVI. In nativitate sancti Iohannis Baptistae II.

- Homilia LXVII. In nativitate sancti Iohannis Baptistae III.

- Homilia LXVIII. In natali beatissimorum apostolorum Petri et Pauli I.

- Homilia LXIX. De eodem natali sanctorum apostolorum Petri et Pauli.

- Homilia LXX. De eodem natali sanctorum apostolorum Petri et Pauli.

- Homilia LXXI. De eodem natali sanctorum apostolorum Patri et Pauli.

- Homilia LXXII. De eodem natali sanctorum apostolorum Petri et Pauli.

- Homilia LXXIV. In natali sancti Laurentii Levitae et Martyris I.

- Homilia LXXVI. De eodem natali III.

- Homilia LXXVIII. De eodem sancto Eusebio Episcopo Vercellensi, II.

- Homilia XC. De poenitentia et ieiuniis Ninivitarum I.

- Sermo XVIII. In dedicatione Ecclesiae I.

- Sermo XXIX. In Paschatis sollemnitate I.

- Sermo XXX. In festo dedicationis Ecclesiae. 
- Sermo LVI. In natali sanctae Agnetis.

Pedro Crisólogo:

- Sermo L. De paralytico curato.

- Sermo CXXVII. De decolatione Iohannis Baptistae.

- Sermo CLII. De Infantium nece.

Rabano Mauro:

- Commentaria in Libros Machabaeorum. In Liber Primum, Cap. IV.

- Commentarium in Mathaeum, Cap. VII, vs. iii.

- De Universo, Liber IV, Cap. Primus.

- Homilia XXXII. In revelatione sancti Michaelis.

- Homilia LXX. Reversio sanctae atque gloriosissima crucis Domini nostri Iesu.

Zenón de Verona:

- Tractatus IV. De pudicitia.

Recebido em: 30 de maio de 2019 Aceito em: 03 de dezembro de 2019 